\title{
Fabrication and electrical characterization of highly ordered copper nanowires
}

\author{
Gurmeet Singh Lotey $\cdot$ Sanjeev Kumar • \\ N. K. Verma
}

Received: 25 August 2011/Accepted: 18 September 2011/Published online: 30 September 2011

(C) The Author(s) 2011. This article is published with open access at Springerlink.com

\begin{abstract}
The template-assisted electrodeposition technique has been employed to synthesize highly ordered: uniformly dense, well-aligned, parallel and homogeneous copper nanowires. Their morphological studies have been carried out using the scanning electron microscopy and transmission electron microscopy. The X-ray diffraction study exhibits cubic structure of the nanowires and their preferred orientation along the direction [111]. Their elemental composition has been done by energy dispersive $\mathrm{X}$-ray analysis. The photoluminiscence spectra of copper nanowires show two excitation peaks at 209 and $268 \mathrm{~nm}$; both these absorption pathways yield fluorescence at $296 \mathrm{~nm}$. Their UV-Vis absorption spectra have been found to give a prominent peak at $570 \mathrm{~nm}$. The current-voltage characteristics of the nanowires reveal their non-linear behavior. The impedance spectroscopy has also been carried out and it shows an increase in their impedance at higher frequencies.
\end{abstract}

Keywords Electrodeposition - Nanowires ·

Nonlinear behavior · Impedance

\section{Introduction}

One-dimensional (1D) nanostructures such as nanotubes, nanowires, nanobelts have been extensively studied not

G. S. Lotey $(\varangle) \cdot$ N. K. Verma

Nano Research Lab, School of Physics and Materials Science,

Thapar University, Patiala 147 004, India

e-mail: gslotey1986@gmail.com; gslotey@thapar.edu

S. Kumar

University College of Engineering, Punjabi University,

Patiala 147 002, India only for their novel physical, chemical, electronic, magnetic, and electrical properties but also for their prospective applications in future-generation nanodevices. Among the various hierarchical structures, 1D nanostructure arrays, perpendicular to the substrate, are of significant interest. The 1D nanostructures are ideal building blocks for nanoelectronics (Liu and Bando 2003) since, in the architecture of nanodevices, they can act both as interconnects and device components. The miniaturization of basic device elements, using different types of junctions, leads to the vast success in microelectronics industry. The primary goal of nanoelectronics industry is to achieve similar functionality at nanoscale. However, the basic problem arises when the essential device elements are joined together by $1 \mathrm{D}$ nanostructures to form multi-terminal nanodevices eventually leading to complex circuits. Thus, the study on highly ordered metal nanowires is of great motivation (Zhang et al. 2005; Zach et al. 2000). The metallic nanowires have potential applications in a wide range of advanced fields (Zhang et al. 2005; Zach et al. 2000; Toma et al. 2010; Mehrez and Guo 2004; Wildoer et al. 1998). Due to accessibility and exceptional properties such as good strength, admirable malleability, and superior corrosion resistance, copper is the third most broadly used commercial metal after iron and aluminum. Furthermore, copper has outstanding electrical and thermal conductivity second to silver. Therefore, copper nanowires are of particular importance. With the fast reduction in size of electronic devices, copper nanowires play a vital role as interconnects in nanoelectronics and optoelectronic devices. The number of methods used for the fabrication of such nanowires, includes vapor deposition (Liu and Bando 2003; Zhang et al. 2005), reverse micelle system (Lisiecki et al. 1996), electrochemical synthesis (Liu et al. 2008; Bicelli et al. 2008; Zhang and Wong 2009), ion-beam 
sputtering (Toma et al. 2010), solution-phase synthesis (Wang et al. 2008), etc. (Choi and Park 2004; Zhang et al. 2008; Duan et al. 2010; Monson and Woolley 2003; Lai and Riley 2008; Qin et al. 2007). An ordinary fabrication method for nanowires is based on the electrodeposition of metals into pores of controlled geometry. Nanostructures synthesized by template-assisted technique, using anodic alumina membrane (AAM) (Furneaux et al. 1989; Li et al. 1998), are quite attractive due to these templates' remarkable hardness, uniform pore size, high pore density together with relatively low cost. The electrodeposition method is influenced by several parameters, e.g., temperature, $\mathrm{pH}$, overvoltage, and composition of the electrolyte, which have been studied for different systems (Gao et al. 2002; I.U. Schuchert et al. 2003; Pan et al. 2005a, 2005b; Pan et al. 2005a, 2005b).

This paper presents synthesis of highly ordered copper nanowires using template-assisted electrodeposition technique. The electrical characterization viz. current-voltage (I-V) characteristics and the impedance spectroscopy of the nanowires are also being studied. The nanowires showed a unique behaviour; they did not obey Ohm's law rather the charge carriers were found to tunnel through a potential barrier; also an increase in impedance was noticed at high frequencies.

\section{Experimental}

The commercially available templates (Anodisc 25, Whatman, UK) with average pore diameter, $100 \mathrm{~nm}$, and pore density, $10^{9} \mathrm{~cm}^{-2}$ have been used for the fabrication of copper nanowires. The chemicals and reagents used were of analytical grade. The de-ionized water was used

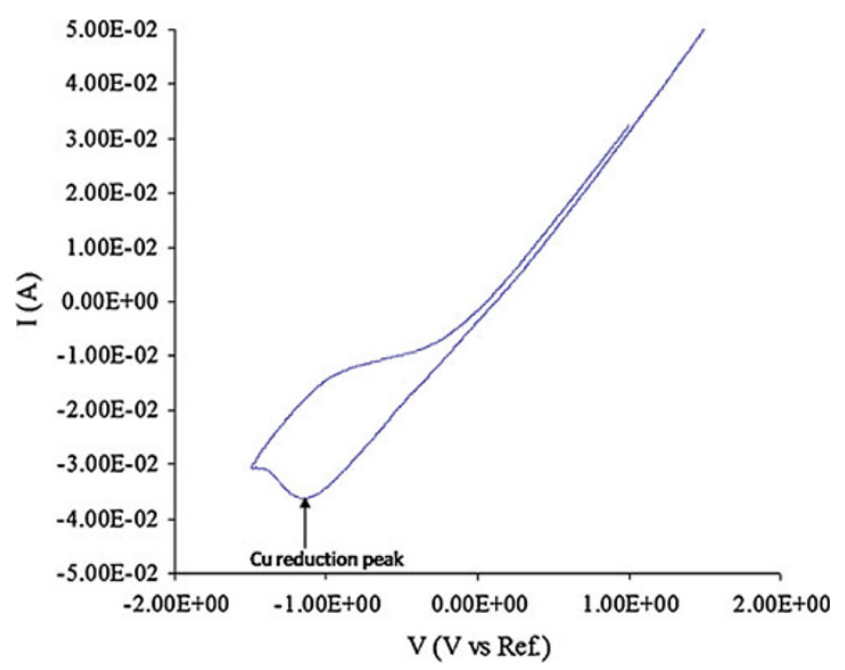

Fig. 1 The cyclic voltammetry curve for copper electrodeposition in anodic alumina membrane for preparing solutions. The electrodeposition process has been carried out in a typical three-electrode electrochemical cell using Potentiostat (Gamry Reference 600) with a platinum electrode as counter electrode, $\mathrm{Ag} / \mathrm{AgCl} \mathrm{C}_{2}$ as reference electrode, and the AAM coated with $\mathrm{Ag}$ as working electrode. $0.1 \mathrm{M} \mathrm{CuSO}_{4} .5 \mathrm{H}_{2} \mathrm{O}$ aqueous solution was used as electrolyte, and the $\mathrm{pH}$ of this solution was adjusted to be 2 using $0.1 \mathrm{M} \mathrm{H}_{2} \mathrm{SO}_{4}$. A high concentration of $\mathrm{CuSO}_{4}$ is required to supply a sufficiently large number of copper ions $\left(\mathrm{Cu}^{2+}\right)$ inside the pores of AAM during the electrodeposition process. $\mathrm{H}_{2} \mathrm{SO}_{4}$ increases the conductivity of the solution and lowers the cathode overvoltage Using a cyclic voltametry curve (Fig. 1), the reduction potential, required for depositing copper, was found to be $1.2 \mathrm{~V}$. By applying this low voltage, side reactions such as evolution of hydrogen bubbles were avoided.

\section{Characterization and measurements}

The crystallographic study of copper nanowires embedded in AAM as host was performed using the X-ray diffractometer (PANalytical, X'Pert PRO MRD ML) with $\mathrm{CuK} \alpha$ ( $\lambda=1.5418 \AA$ Á) radiation operated at $45 \mathrm{kV}$ and $40 \mathrm{~mA}$. The high intense beam was focused over a small area $\left(10 \mathrm{~mm}^{2}\right)$ of the sample, and gonio scan was recorded for $2 \theta$ values from $10^{\circ}$ to $90^{\circ}$. The KEITHLEY source meter, 4200-SCS, attached to ZYVEX S100 Nanomanipulator, was used for collective I-V measurements. The impedance spectroscopy was carried out using Gamry Reference 600 potentiostat and an especially designed Faraday cage at frequencies from $0.2 \mathrm{~Hz}$ to $1 \mathrm{MHz}$. For scanning electron microscopy (SEM) characterization, the prepared sample of copper nanowires embedded in the AAM was put in a solution of $\mathrm{NaOH}$ to dissolve the alumina membrane. A very small concentration $(0.01 \mathrm{M})$ of $\mathrm{NaOH}$ was used to prevent damage of the nanowires. The nanowires were then washed several times with de-ionized water followed by ethanol. A small drop of the above solution, containing the nanowires, was taken for the SEM and TEM characterizations. For SEM analysis, the dried sample of copper nanowires was coated with goldpalladium alloy using JEOL, JFC sputter coater and then examined under JEOL, JSM-6510 L scanning electron microscope. The energy dispersive X-ray spectroscopy (EDAX) was used for elemental composition analysis using Noran System Six attached to SEM. The transmission electron microscopy (TEM) measurements were taken with a Hitachi H-7500 system operating at $110 \mathrm{kV}$. The optical characterization of copper nanowires has been done by UVVis spectroscopy using UV-Thermoevolution spectrometer. The photoluminescence (PL) study has been carried out using Cary Varian fluorospectrophotometer with xenon lamp as an excitation source. 


\section{Results and discussion}

Growth and morphology study

Figure 2a and $\mathrm{b}$ show the SEM micrographs respectively for the cross-sectional and lateral view of the nanowires showing their dense growth. They are found to be uniformly dense, homogeneous, parallel, and well aligned. The nanowires are found to be of size, $100 \mathrm{~nm}$, and length, $12 \mu \mathrm{m}$ as confirmed by TEM (Fig. 3). The aspect ratio of the nanowires is about 120 .

The nucleation and growth inside the nanochannels of AAM result in the formation of copper nanowires; their growth is based on the reduction of $\mathrm{Cu}^{2+}$ ions by electrodeposition as shown below:

$\mathrm{CuSO}_{4} \rightarrow \mathrm{Cu}^{2+}+\mathrm{SO}^{4-}$

The $\mathrm{Cu}^{2+}$ ions are introduced into the nanochannels. On applying the electric field, they get reduced on the surface of the working electrode as follows:

$\mathrm{Cu}^{2+} \rightarrow \mathrm{Cu}+2 \mathrm{e}^{-}$

First, the $\mathrm{Cu}$ nuclei are formed at the base of the template, and then the copper nanowires grow along the pores because of the trapping effect of the template. Their growth is controlled by the parameters such as applied potential, concentration of ions, flow rate of the electroactive species and $\mathrm{pH}$ value of the solution.

\section{Structural study}

The X-ray diffraction (XRD) analysis of the copper nanowires, embedded in AAM, has been carried out to study crystal structure (Fig. 4). It shows the presence of the four reflection peaks attributed to planes, (llll 111$),\left(\begin{array}{lll}2 & 0 & 0\end{array}\right),(2$ $20)$, (3 11 1), confirming copper nanowires with the facecentered cubic crystal structure. From Fig. 4, it is also clear

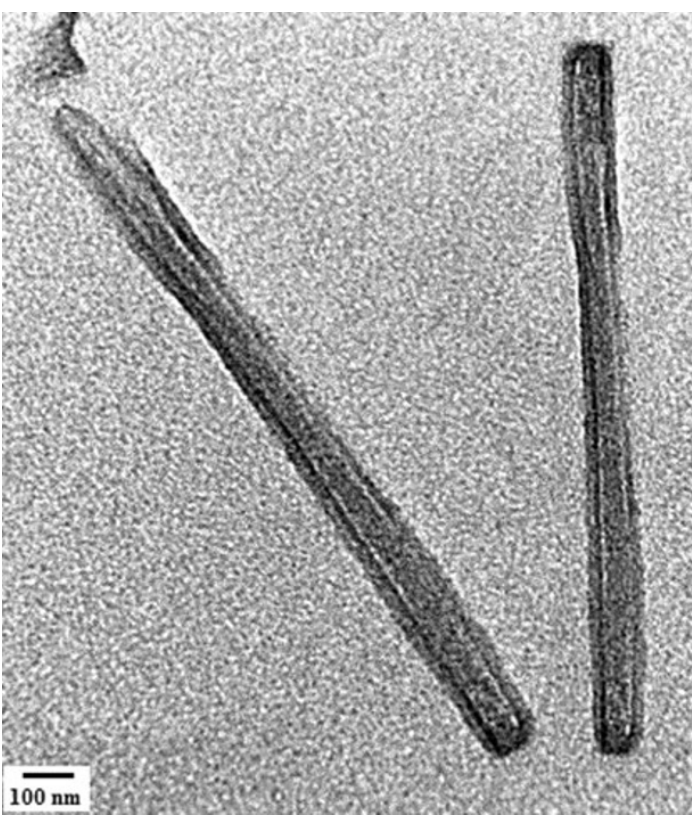

Fig. 3 The transmission electron microscopy (TEM) images of copper nanowires

that the growth of copper nanowires is preferably along the direction, [111]. The reflection peaks observed for silver may be due to some silver paste still left at the AAM. The EDAX spectrum of copper nanowires (Fig. 5) exhibits the peaks of copper along with those of gold; as the latter is required for SEM. Some small peaks due to environmental carbon and oxygen are also observed.

Optical study

\section{Photoluminescence study}

Two peaks at 268 and $209 \mathrm{~nm}$, corresponding to maximum transition probability/resonant absorption, have been
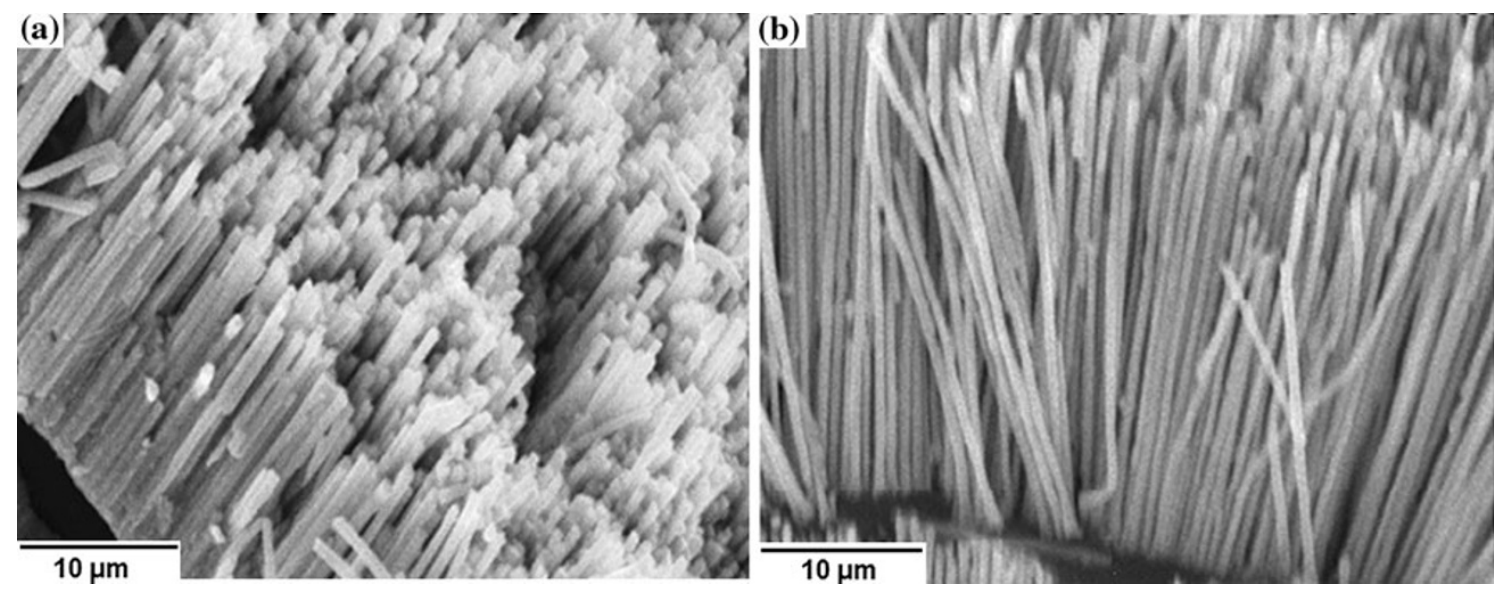

Fig. 2 The SEM micrographs of copper nanowires

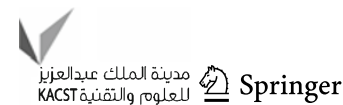




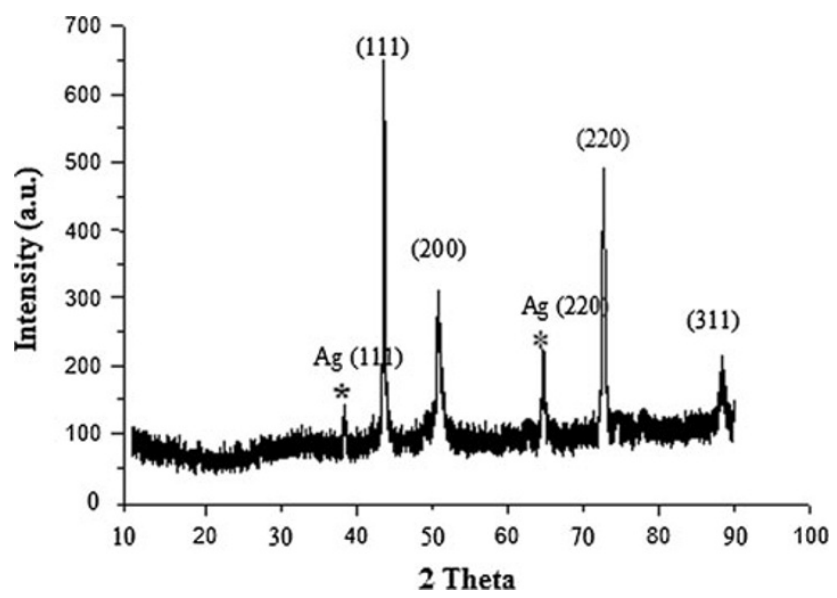

Fig. 4 The XRD pattern of copper nanowires

observed (Fig. 6a) in the fluorescence excitation spectra; these peaks correspond, respectively, to the transition between the ground state $\left(\mathrm{E}_{0}\right)$ and the first excited state $\left(E_{1}\right)$, and the transition between the ground state and the second excited state $\left(E_{2}\right)$. Both of these absorption pathways have been found to yield fluorescence at $296 \mathrm{~nm}$. Both the excitation wavelengths, 209 and $268 \mathrm{~nm}$, yield the fluorescence emission at $296 \mathrm{~nm}$ (Fig. 6b); this emission peak has been found to remain fixed whatever the excitation wavelengths. The fluorescence from noble metals has been attributed to transitions of electrons in the conduction band below the Fermi level to the holes in the $d$ bands (Mooradian 1968; Boyd et al. 1986). The peak observed at $296 \mathrm{~nm}$ in fluorescence spectra is due to transitions from the excited states to $d$-levels of the copper nanowires (Siwach and Sen 2008). However, the intensity of fluorescence peaks varies with change in $\lambda_{\mathrm{ex}}$ (Fig. 6b) because of the change in transition probability.

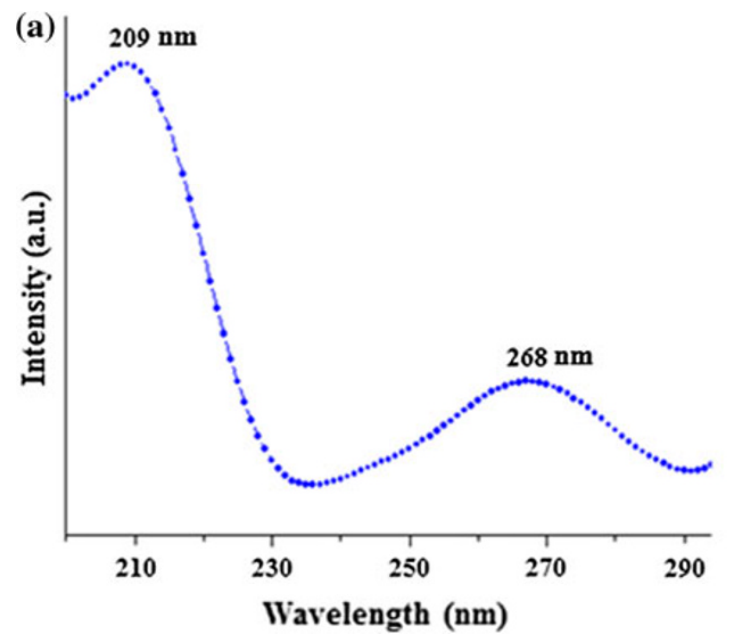

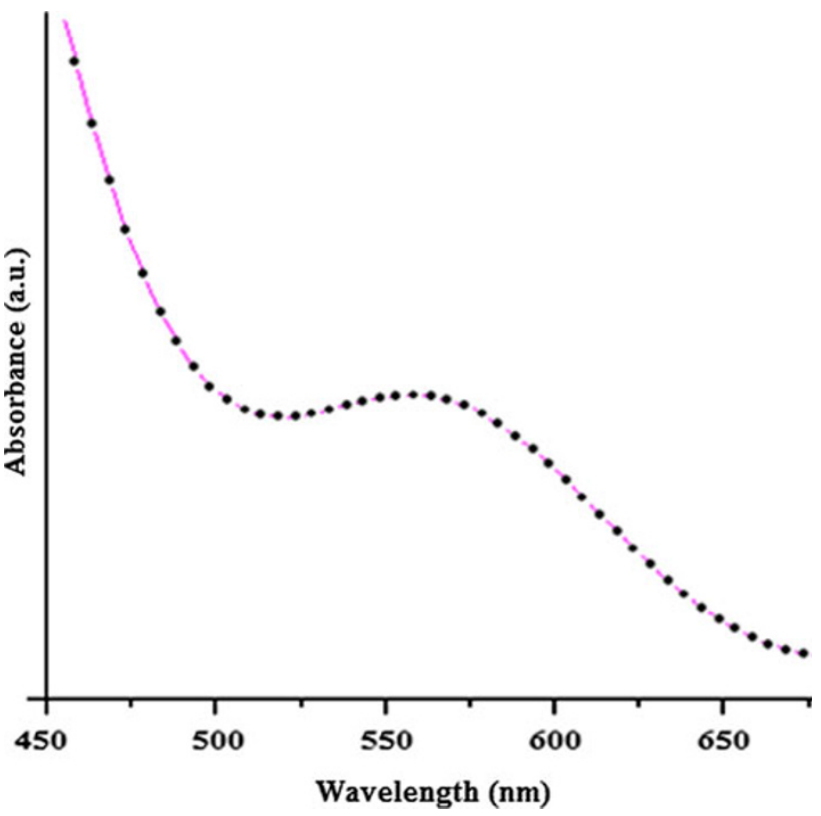

Fig. 6 UV-vis absorption spectrum of copper nanowires

\section{$U V$-visible analysis}

The metal nanoparticles exhibit absorption bands or broad regions of absorption in the UV-visible range due to the excitation of surface plasmon resonances (SPR) or interband transitions; these SPR are characteristic properties for the metallic nature of particles. The prominent peak observed at $570 \mathrm{~nm}$, in the visible wavelength, is due to the absorption of surface plasmon (Fig. 7). This broadband absorption, in the copper nanowires, is attributed to SPR and its tail at wavelength $510 \mathrm{~nm}$, to $d-s p$ interband transitions. The bandwidth of the resonance increases due to the

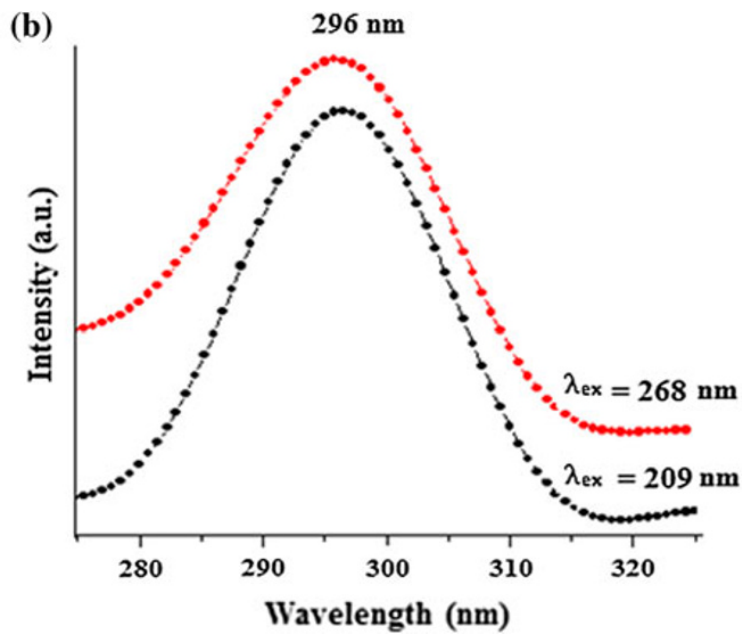

Fig. 5 a The fluorescence excitation spectra of copper nanowires and $\mathbf{b}$ fluorescence emission spectra at excitation wavelengths 209 and $268 \mathrm{~nm}$ 
Fig. 7 The EDAX spectrum of copper nanowires

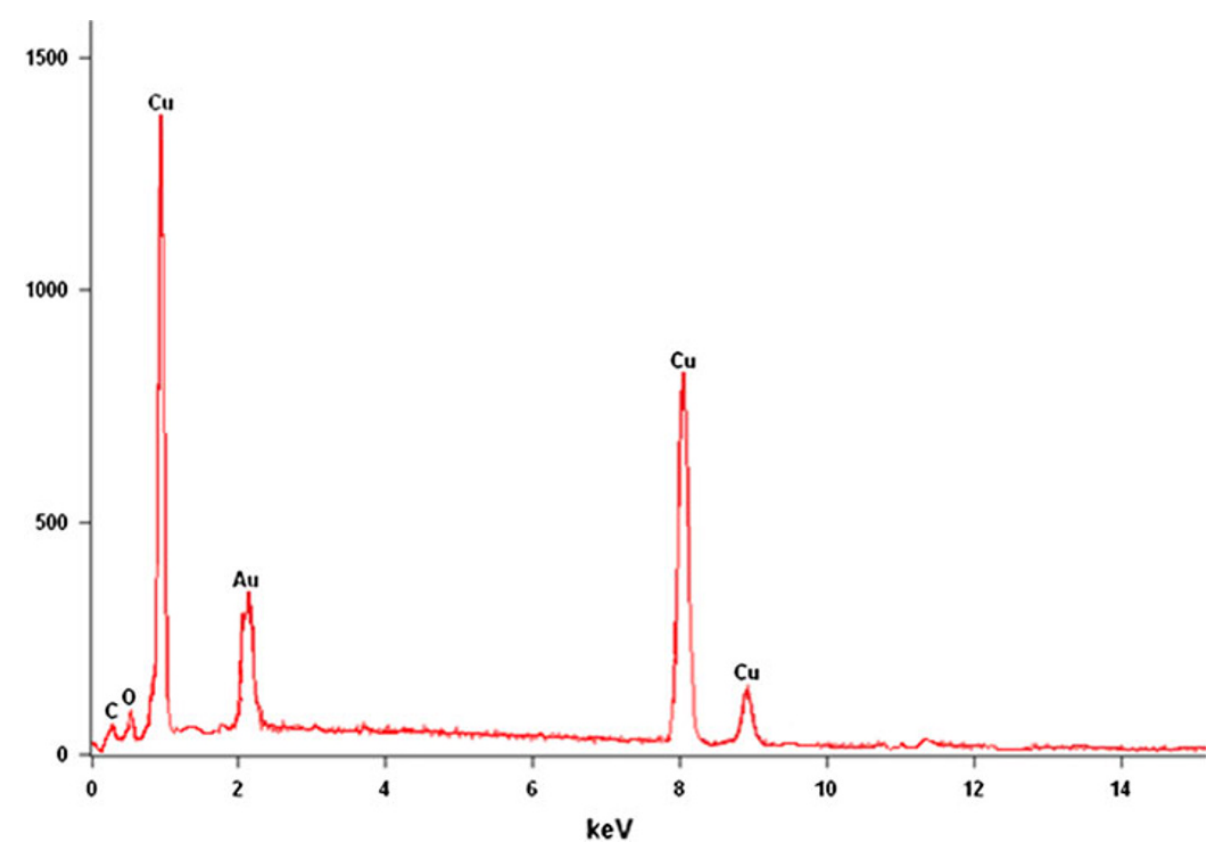

(a)

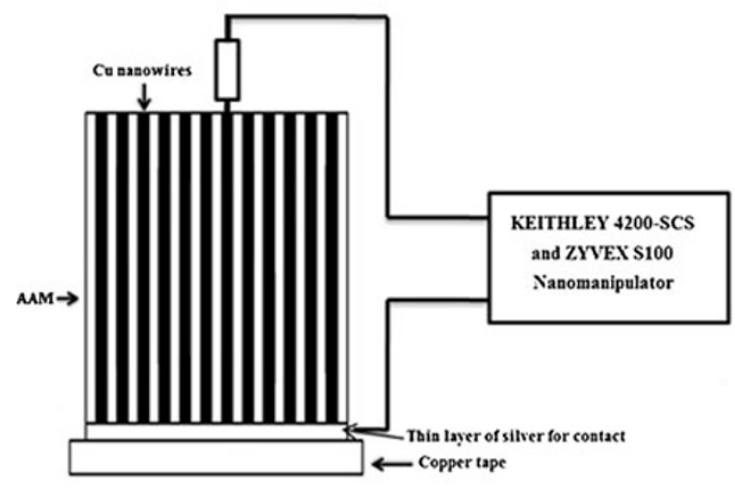

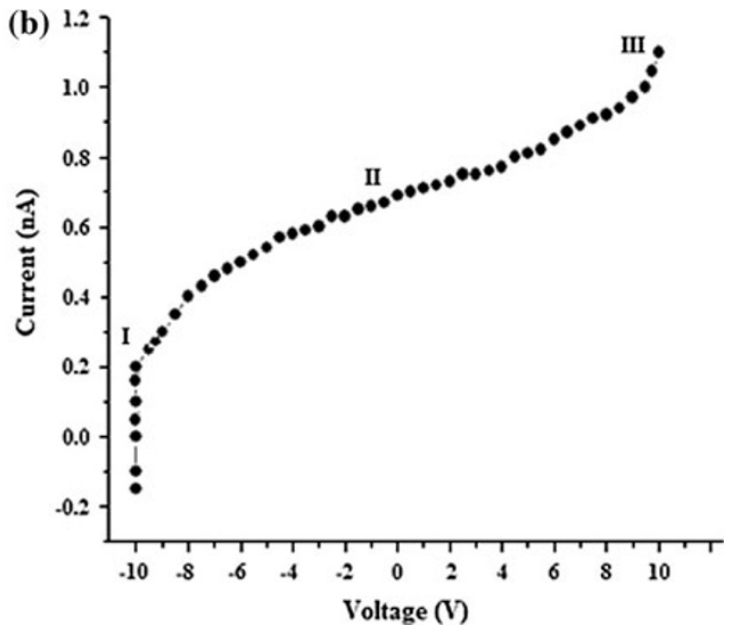

Fig. 8 a The schematic of collective $\mathrm{I}-\mathrm{V}$ characteristics measurement set up and b I-V characteristics curve of copper nanowires

decrease in size of the particles as well as the enhancement in the scattering of electrons at the surface of nanowires.

Electrical and transport properties

\section{Current voltage characteristics}

Figure 8a shows the schematic set up for the measurement of collective I-V characteristics of copper nanowires. Figure $8 \mathrm{~b}$ displays their non-linear and symmetric characteristics, with respect to zero bias. This exhibits a very unique behaviour of the nanowires that they do not obey the Ohm's law rather the electrons, the charge carriers, are being tunneled through a potential barrier. The $\mathrm{I}-\mathrm{V}$ curve can
Table 1 The measurement of voltage (V), current (I), resistance (R), and conductance, (S) corresponding to segments I, II, III of I-V characteristics

\begin{tabular}{llllll}
\hline S. no & Region & $\begin{array}{l}\text { Voltage } \\
(\mathrm{V})\end{array}$ & $\begin{array}{l}\text { Current } \\
(\mathrm{nA})\end{array}$ & \multicolumn{1}{l}{$\begin{array}{l}\text { Resistance } \\
(\Omega)\end{array}$} & \multicolumn{1}{l}{$\begin{array}{l}\text { Conductance } \\
(\mathrm{S})\end{array}$} \\
\hline 1 & I & -10 & 0.3 & $33.33 \times 10^{9}$ & $0.30 \times 10^{-10}$ \\
2 & II & -1.0 & 0.7 & $1.43 \times 10^{9}$ & $6.99 \times 10^{-10}$ \\
3 & III & 10 & 1.0 & $10 \times 10^{9}$ & $1.0 \times 10^{-10}$ \\
\hline
\end{tabular}

be divided into three different segments, i.e., I, II, and III corresponding to the conductance values $0.3 \times 10^{-10}$, $6.99 \times 10^{-10}, 1.0 \times 10^{-10} \mathrm{~S}$, respectively; this data further 
Fig. 9 The impedance versus frequency behavior of copper nanowires

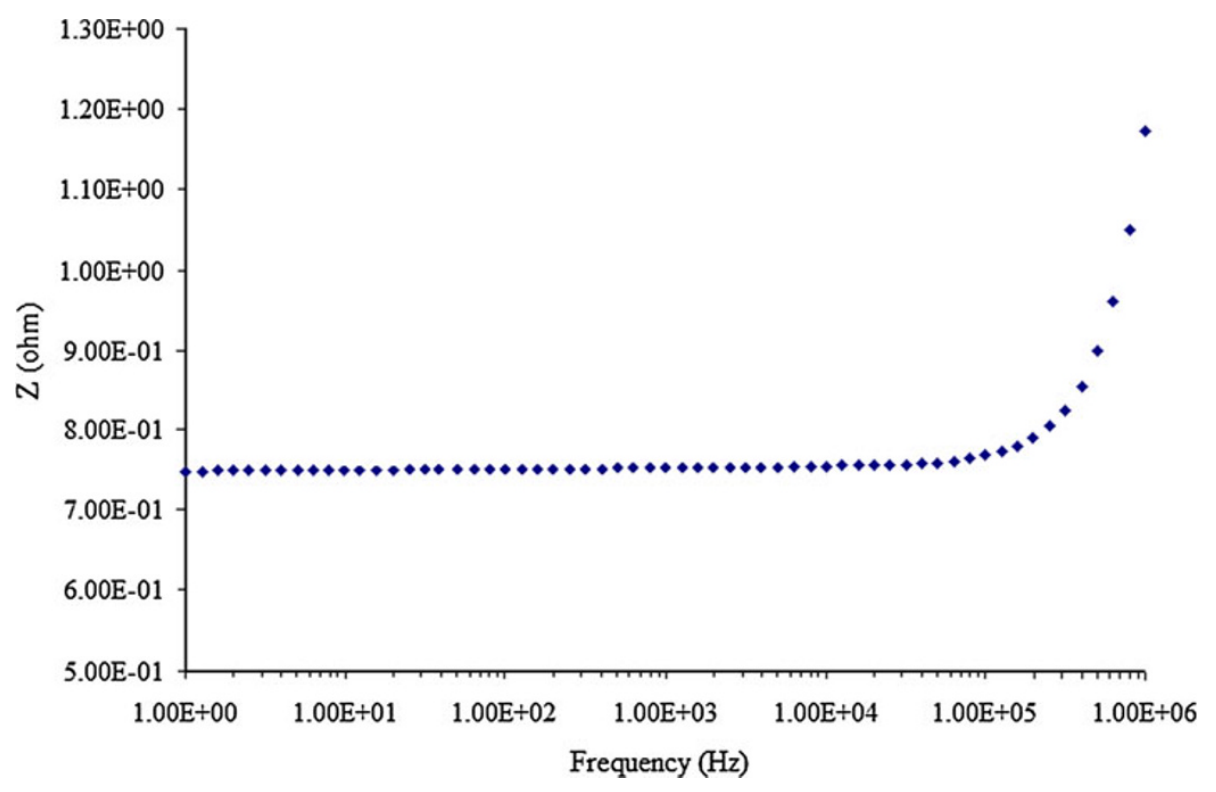

confirms the tunneling behaviour. The tunneling behaviour may be due to the contact potential between the nanowires and the probe; this (the contact potential) being a function of voltage (Mehrez and Guo 2004). Similar non-linear I-V behaviour has been observed for silver (Mehrez and Guo 2004), and gold (Wildoer et al. 1998) nanowires; such a behavior is required for functional electronic devices (Itakura et al. 1999). Figure 8b shows a quasi-plateau formation in region II, also observed by Cao et al. (2006), and the sharp increase and decrease in current noticed for the forward as well as the reverse bias correspond, respectively, to around $+8.8 \mathrm{~V}$ and $-9.4 \mathrm{~V}$. For the three segments, the voltage, current, resistance and conductance are shown in Table 1.

\section{Impedance analysis}

Figure 9 stands for the impedance spectroscopy of the nanowires, embedded in AAM as host, at frequencies 0.2-1.0 MHz. An increase in impedance has been observed after frequency, $1 \mathrm{kHz}$, confirming inductive effect, which may be due to the mutual induction among the copper nanowires.

\section{Conclusions}

We have synthesized highly ordered and dense crop of copper nanowires using a template-assisted electrodeposition technique; the nanowires have been found to be of average size, $100 \mathrm{~nm}$, and preferentially grown along the direction, [111]. The absorption spectra reveal that the peak, observed at $570 \mathrm{~nm}$, is due to absorption of surface plasmon. The two excitation peaks, observed at 209 and
$268 \mathrm{~nm}$, yield a fluorescence peak at $296 \mathrm{~nm}$ in PL spectra, which is due to the fluorescence emission from the copper nanowires at different excitation wavelength..The nonlinear I-V characteristics suggest formation of tunneling potential barrier in copper nanowires. The nanowires register rise in impedance above $1 \mathrm{kHz}$ due to the inductive effect. The nonlinear I-V characteristics clearly show the possibility of the synthesized copper nanowires finding potential applications in future nanoelectronics. Moreover, these nanowires, being highly-ordered, may be directly patterned for fabrication of future nanodevices.

Acknowledgments Gurmeet Singh Lotey is extremely grateful to the Department of Science and Technology, India, for awarding him the INSPIRE fellowship to carry out the present research work.

Open Access This article is distributed under the terms of the Creative Commons Attribution License which permits any use, distribution and reproduction in any medium, provided the original author(s) and source are credited.

\section{References}

Bicelli LP, Bozzini B, Mele C, Durzo L (2008) A review of nanostructural aspects of metal electrodeposition. Int J Electrochem Sci 3:356

Boyd GT, Yu ZH, Shen YR (1986) Photoinduced luminescence from the noble metals and its enhancement on roughened surfaces. Phys Rev B 33:7923

Cao H, Wang L, Qiu Y, Zhang L (2006) Synthesis and I-V properties of aligned copper nanowires. Nanotechnology 17:1736

Choi H, Park SH (2004) Seedless growth of free-standing copper nanowires by chemical vapor deposition. J Am Chem Soc 126:6248

Duan J, Liu J, Mo D, Yao H, Maaz K, Chen Y, Sun Y, Hou M, Qu X, Zhang L, Chen Y (2010) Controlled crystallinity and 
crystallographic orientation of $\mathrm{Cu}$ nanowires fabricated in iontrack templates. Nanotechnology 21:365605

Furneaux RC, Rigby WR, Davidson AP (1989) The formation of controlled-porosity membranes from anodically oxidized aluminium. Nature 337:147

Gao T, Meng GW, Wang Y, Sun S, Zhang LD (2002) Electrochemical synthesis of copper nanowires. J Phys Condens Matt 14:355

Itakura K, Yuki K, Kurokawa S, Yasuda H, Sakai A (1999) Bias dependence of the conductance of Au nanocontacts. Phys Rev B 160:1163

Lai M, Riley DJ (2008) Templated electrosynthesis of nanomaterials and porous structures. J Coll Int Sci 323:203

Li AP, Muller F, Birner A, Nielsch K, Gosele U (1998) Hexagonal pore arrays with a $50-420 \mathrm{~nm}$ interpore distance formed by selforganization in anodic alumina. J Appl Phys 84:6023

Lisiecki I, Filankembo A, Kongehl HS, Weiss K, Pileni MP (1996) Control of the shape and the size of copper metallic particles. J Phys Chem 100:4160

Liu Z, Bando Y (2003) A novel method for preparing copper nanorods and nanowires. Adv Mater 15:303

Liu N, Wu D, Wu H, Liu C, Luo F (2008) A versatile and "green" electrochemical method for synthesis of copper and other transition metal oxide and hydroxide nanostructures. Mater Chem Phys 107:511

Mehrez H, Guo H (2004) In: Wang Zl (ed) Nanowires and nanotubesmaterials, properties and devices-metal and semiconductor nanowires, vol 1. Springer, Berlin

Monson CF, Woolley AT (2003) DNA-templated construction of copper nanowires. Nano Lett 3:359

Mooradian A (1968) Photoluminescence of metals. Phys Rev Lett 22:185

Pan H, Sun H, Poh C, Feng Y, Lin J (2005a) Single-crystal growth of metallic nanowires with preferred orientation. Nanotechnology $16: 1559$
Pan H, Liu B, Yi J, Poh C, Lim S, Ding J, Feng Y, Huan CHA, Lin J (2005b) Growth of single-crystalline Ni and Co nanowires via electrochemical deposition and their magnetic properties. J Phys Chem B 109:3094

Qin Y, Staedler T, Jiang X (2007) Preparation of aligned Cu nanowires by room-temperature reduction of $\mathrm{CuO}$ nanowires in electron cyclotron resonance hydrogen plasma. Nanotechnology 18:35608

Schuchert IU, Toimil Molares ME, Dobrev D, Vetter J, Neumann R, Martin M (2003) Electrochemical copper deposition in etched ion track membranes. J Electrochem Soc C150:C189

Siwach OP, Sen P (2008) Fluorescence properties of Ag nanoparticles in water, spectrochimca acta part A. Mol Biomol Spectrosc 69:659

Toma A, Chiappe D, Boragno C, Mangeot FB (2010) Self-organized ion-beam synthesis of nanowires with broadband plasmonic functionality. Phys Rev B 81:165436

Wang C, Hu YJ, Lieber CM, Sun SH (2008) Facile synthesis of ultrathin and single-crystalline Au nanowires. J Am Chem Soc 130:8902

Wildoer JWG, Venema LC, Rinzler AG, Smalley RE, Dekker C (1998) Electronic structure of atomically resolved carbon nanotubes. Nature 391:59

Zach MP, Ng KH, Penner RM (2000) Molybdenum nanowires by electrodeposition. Science 290:2120

Zhang F, Wong SS (2009) Controlled synthesis of semiconducting metal sulfide nanowires. Chem Mater 21:4541

Zhang J, Sun J, Wei W, Shi S, Sun H, Guo J (2005) Synthesis of copper nanowires under a direct current electric field. Nanotechnology 16:2030

Zhang DW, Chen CH, Zhang J, Ren F (2008) Fabrication of nanosized metallic copper by electrochemical milling process. J Mater Sci 43:1492 